\title{
The contribution of stereo vision to the control of braking
}

\author{
Pieter Tijtgat ${ }^{\mathrm{a}}$, Liesbeth Mazyn ${ }^{\mathrm{a}}$, Christophe De Laey ${ }^{\mathrm{b}}$, Matthieu Lenoir ${ }^{\mathrm{a}}$ \\ ${ }^{a}$ Department of Movement and Sports Sciences, Faculty of Medicine and Health Sciences, \\ Ghent University, Watersportlaan 2, B-9000 Ghent, Belgium \\ ${ }^{b}$ Department of General physiology and human physiology and pathophysiology, Faculty of Medicine \\ and Health Sciences, Ghent University, De Pintelaan 185, B-9000 Ghent, Belgium \\ Telephone: +32-(0)92649137 \\ Fax: +32-(0)92646448 \\ Email: pieter.tijtgat@ugent.be
}

\section{Introduction}

To be successful in the confusion of daily traffic, executing appropriate brake manoeuvres is an important skill. When following a car, a driver needs to be alert in order to quickly register and act upon decelerations of a leading vehicle (1). From the literature (2-3), it can be concluded that monocular information sources like the optical variable tau play an important role in the visual control of braking, but that several other visual cues, such as depth information from stereo vision, might have a supplement impact on brake behaviour. In present experiment, the contribution of stereo vision was investigated when performing a braking task

\section{Methods}

26 female participants with normal ( $\leq 40$ arcsec on a Grade Circle Test; StereoN; N $=13$ ) or weak $(\geq 800 \mathrm{arcsec}$; StereoW; $N=13)$ stereo vision performed braking tasks when driving at a speed of approximately $11 \mathrm{~km} / \mathrm{h}$ on a go-cart. Subjects could start braking at 4,7 or 10 meters from a target vehicle when a red lamp on the rear of this vehicle lit. The momentanuous position of the go-cart was measured at $200 \mathrm{~Hz}$ with a laser (Noptel CMP2-30) and velocity and acceleration were calculated. From these data, time and distance of brake initiation, and time of maximal deceleration were calculated as principal dependent variables and submitted to a 3 (conditions of distance: $4 \mathrm{~m}$ vs. $7 \mathrm{~m}$ vs. $10 \mathrm{~m})$ x 2 (group conditions: stereoW, stereoN) ANOVA with repeated measures on the first factor.

\section{Results}

StereoW group started braking earlier in time than StereoN $(\mathrm{p} \leq .05)$. In the braking adjustment phase, maximal deceleration occurred closer in time to contact for StereoN group than StereoW ( $\mathrm{p}<.01)$. This effect increased up to $2596 \mathrm{msec}$ in the 10m-condition, as the interaction effect showed up $(\mathrm{p}<.01)$. StereoN group reached standstill on a greater distance than StereoW group $(\mathrm{p}<.05)$.

\section{Conclusions}

From this experiment, it can be concluded stereo vision contributes to the initiation as well as to the control of a braking manoeuvre. However, this does not mean that people with a lack of stereo vision may cause greater risks in traffic negotiation, in particular situations in which rear-end collisions may occur. The lack of good stereo vision is even associated with more prudent brake behaviour, i.e. an earlier brake initiation and an earlier maximal deceleration.

\section{Key References}

1. Fajen, B. R. (2005). The scaling of information to action in visually guided braking. Journal of Experimental Psychology-Human Perception and Performance, 31, 1107-1123

2. Lee, D. N. (1976). Theory of Visual Control of Braking based on Information about Time-ToCollision. Perception, 5, 437-459

3. Tresilian, J. R. (1991). Empirical and Theoretical Issues in the Perception of Time To Contact. Journal of Experimental Psychology-Human Perception and Performance, 17, 865-876 